\title{
EXACT TRAVELLING WAVE SOLUTIONS FOR THE GENERALIZED KURAMOTO-SIVASHINSKY EQUATION
}

\section{JIAN YANG, XIAOJUAN LU and SHENGQIANG TANG}

School of Mathematics and Computing Science

Guilin University of Electronic Technology

Guilin, Guangxi, 541004

P. R. China

e-mail: tangsq@guet.edu.cn

\begin{abstract}
By using transformation $u^{\prime \prime}=a_{1}+a_{2} u+a_{3} u^{2}$, the method of sine-cosine and the method of dynamical bifurcation theory of the differentiable dynamics, we study the generalized Kuramoto-Sivashinsky equation. It is shown that the generalized Kuramoto-Sivashinsky equation gives solitary wave solution, solitary patterns wave solution, and periodic wave solution. Under different parametric conditions, various sufficient conditions to guarantee the existence of the above solutions are given. All exact explicit parametric representations of the above waves are determined.
\end{abstract}

2010 Mathematics Subject Classification: 35C08, 37B55.

Keywords and phrases: solitary wave, periodic wave, solitary patterns wave, method of sinecosine, method of dynamical bifurcation theory of the differentiable dynamics, generalized Kuramoto-Sivashinsky equation.

This research was supported by NNSF of China (Nos. 11361017 and 11161013) and Natural Science Foundation of Guangxi (No. 2013GXNSFAA019010).

Received November 28, 2014

(ㄷ) 2015 Scientific Advances Publishers 


\section{Introduction}

In recent years, much attention has been paid on the study of nonlinear wave equations (NLWE) in low dimensions. But there is little work on the high dimensional ones. It is well known that most of high dimensional NLWEs fail the conventional integrability tests, so the natural and important problem is that are there exact solutions with good properties for the high dimensional NLWEs? To solve this problem, people have made some attempts.

In 1990, Aspe and Depassier [1] considered the following evolution equation of surface waves in a convecting fluid:

$$
u_{t}+\lambda_{1} u u_{x}+\lambda_{2} u_{x x x}+\epsilon\left[\frac{\sigma R_{2}}{15} u_{x x}+\lambda_{3} u_{x x x x}+\lambda_{4}\left(u u_{x}\right)_{x}\right]=0
$$

where $\sigma$ is the Prandtl number and $\epsilon$ is a small parameter such that the excess of the Rayleigh number above its critical value is given by $\epsilon^{2} R_{2}$. The coefficients $\lambda_{i}, i=1-4$, are functions of the parameters of the problem. Subscripts denote derivatives with respect to the time $t$ and horizontal coordinate $x$. This equation without the effect of instability, that is, with $R_{2}=0$, has been found recently to be the generic equation that describes the evolution of marginally diffusively stable wave trains [2]. A similar equation but with $\lambda_{4}=0$ and with instability and diffusion of the same order as dispersion arises in the study of fluid flow along an inclined plane. Numerical studies of this equation have shown that in the dispersion-dominated regime, for periodic boundary conditions, regular arrangements of soliton like pulses appear. The overall evolution of the system for a sufficiently long periodicity interval is apparently governed by the interaction of these pulses.

For $\lambda_{1}=\lambda_{3}=\lambda_{4}=1, \lambda_{2}=\sigma$, and $\frac{\epsilon \sigma R_{2}}{15}=1$, Equation (1.1) becomes to

$$
u_{t}+u u_{x}+u_{x x}+\sigma u_{x x x}+u_{x x x x}+\epsilon\left(u u_{x}\right)_{x}=0 .
$$


Equation (1.2) is called generalized Kuramoto-Sivashinsky (KS) equations [3] and by using the tanh-function method, find explicit solitary travelling wave solutions of Equation (1.2)

In this paper, by using transformation

$$
u^{\prime \prime}(\xi)=a_{1}+a_{2} u(\xi)+a_{3} u^{2}(\xi), \quad \xi=x-c t,
$$

the method of sine-cosine and the method of dynamical bifurcation theory of the differentiable dynamics to Equation (1.2), the existence of solitary wave solution, solitary patterns wave solution, and uncountably infinite many smooth periodic wave solutions is obtained. Under different parametric conditions, various sufficient conditions to guarantee the existence of the above solutions are given. All exact explicit parametric representations of the above waves are determined. The two methods will be described briefly, where details can be found in [4-6] and the references therein.

Let $u(x, t)=u(\xi), \xi=x-c t$, where $c$ is the wave speed. Then (1.2) becomes to

$$
-c u^{\prime}+u u^{\prime}+u^{\prime \prime}+\sigma u^{\prime \prime \prime}+u^{(4)}+\epsilon\left(u u^{\prime}\right)^{\prime}=0 .
$$

Integrating (1.4) once and setting integration constants as $g$, we have

$$
-c u+\frac{1}{2} u^{2}+u^{\prime}+\sigma u^{\prime \prime}+u^{\prime \prime \prime}+\epsilon\left(u u^{\prime}\right)=g .
$$

Substitute (1.3) into Equation (1.5), we have

$$
\sigma a_{1}-g+\left(a_{2} \sigma-c\right) u+\left(\frac{1}{2} a_{3} \sigma\right) u^{2}+\left(1+a_{2}\right) u^{\prime}+\left(\epsilon+2 a_{3}\right) u u^{\prime}=0 .
$$

We know the Equation (1.6) was established, for $a_{1}=-\frac{g}{c}, a_{2}=-1$, $a_{3}=\frac{1}{2 c}, \sigma=-c$, and $\epsilon=\frac{1}{c}$. Therefore, we only consider Equation (1.3). 
The paper is organized as follows. In Section 2, the method of dynamical bifurcation theory of the differentiable dynamics and the sine-cosine method are briefly discussed. In Section 3, represents exact analytical solutions of Equation (1.3) by using the sine-cosine method. In Section 4, we discuss bifurcations of phase portraits of Equation (1.3). In Section 5, all explicit parametric representations of travelling wave solutions are given. In Section 6, the existence of smooth solitary wave solutions and uncountable infinite many non-smooth periodic wave solutions of (1.2) is discussed. In the last section, we conclude the paper and give some discussions.

\section{Analysis of the Two Methods}

The sine-cosine method and the method of dynamical bifurcation theory of the differentiable dynamics have been applied for a wide variety of nonlinear problems. The main features of the two methods will be reviewed briefly.

For both methods, we first use the wave variable $\xi=x-c t$ to carry a $\mathrm{PDE}$ in two independent variables

$$
P\left(u, u_{t}, u_{x}, u_{t t}, u_{x t}, u_{x x}, \ldots, u_{x x x}, \ldots\right)=0,
$$

into an ODE

$$
Q\left(u, u^{\prime}, u^{\prime \prime}, u^{\prime \prime \prime}, \ldots\right)=0 .
$$

Equation (2.2) is then integrated as long as all terms contain derivatives, where integration constants are considered zeros.

2.1. The sine-cosine method admits the use of the solution in the form

$$
u(\xi)= \begin{cases}\lambda \cos ^{\beta}(\mu \xi), & |\mu \xi|<\frac{\pi}{2}, \\ 0, & \text { otherwise }\end{cases}
$$

or in the form

$$
u(\xi)= \begin{cases}\lambda \sin ^{\beta}(\mu \xi), & |\mu \xi|<\pi \\ 0, & \text { otherwise }\end{cases}
$$


where $\lambda, \mu$, and $\beta$ are parameters that will be determined. We therefore use

$$
\begin{gathered}
u(\xi)=\lambda \cos ^{\beta}(\mu \xi), \\
u^{2}(\xi)=\lambda^{2} \cos ^{2 \beta}(\mu \xi), \\
(u)^{\prime \prime}=-\mu^{2} \beta^{2} \lambda^{2} \cos ^{\beta}(\mu \xi)+\mu^{2} \lambda^{2} \beta(\beta-1) \cos ^{\beta-2}(\mu \xi),
\end{gathered}
$$

and for (2.4), we use

$$
\begin{gathered}
u(\xi)=\lambda \sin ^{\beta}(\mu \xi), \\
u^{2}(\xi)=\lambda \sin ^{2 \beta}(\mu \xi), \\
(u)^{\prime \prime}=-\mu^{2} \beta^{2} \lambda^{2} \sin ^{\beta}(\mu \xi)+\mu^{2} \lambda^{2} \beta(\beta-1) \sin ^{\beta-2}(\mu \xi) .
\end{gathered}
$$

We substitute (2.3) or (2.4) into the reduced ordinary differential equation obtained above in (2.2), balance the terms of the cosin functions when (2.3) is used, or balance the terms of the sine functions when (2.4) is used, and solving the resulting system of algebraic equations by using the computerized symbolic calculations to obtain all possible values of the parameters $\lambda, \mu$, and $\beta$.

2.2. The method of dynamical bifurcation theory of the differentiable dynamics.

The standard method of dynamical bifurcation theory of the differentiable dynamics introduced in [4] and the references therein.

\section{Using the Sine-Cosine Method}

Substituting (2.3) into (1.3) yields

$$
-\lambda^{2} \beta^{2} \mu^{2} \cos ^{\beta}(\mu \xi)+\lambda^{2} \mu^{2} \beta(\beta-1) \cos ^{\beta-2}(\mu \xi)=-\lambda \cos ^{\beta}(\mu \xi)+\frac{\lambda^{2}}{2 c} \cos ^{2 \beta}(\mu \xi) .
$$

(3.1) is satisfied only if the following system of algebraic equations holds: 


$$
\beta-1 \neq 0, \quad 2 \beta=\beta-2, \quad-\lambda=-\lambda^{2} \beta^{2} \mu^{2}, \quad \lambda^{2} \mu^{2} \beta(\beta-1)=\frac{\lambda^{2}}{2 c}
$$

Solving the system (3.2) gives

$$
\beta=-2, \quad \mu^{2}=\frac{1}{12 c}, \quad \lambda=3 c .
$$

The results (3.3) can be easily obtained if we also use the sine method (2.4). Combining (3.3) with (2.3) and (2.4), the following periodic solution:

$$
\begin{aligned}
& u_{1}(x, y, t)=3 c \sec ^{2}\left(\frac{1}{6} \sqrt{\frac{3}{c}}(x-c t)\right), \quad c>0, \\
& u_{2}(x, y, t)=3 c \csc ^{2}\left(\frac{1}{6} \sqrt{\frac{3}{c}}(x-c t)\right), \quad c>0
\end{aligned}
$$

are readily obtained.

However, for $c<0$, we obtain the following solitary wave solutions and solitary patterns wave solutions:

$$
\begin{aligned}
& u_{3}(x, y, t)=3 c \operatorname{sech}^{2}\left(\frac{1}{6} \sqrt{\frac{3}{-c}}(x-c t)\right), \quad c<0, \\
& u_{4}(x, y, t)=-3 c \operatorname{csch}^{2}\left(\frac{1}{6} \sqrt{\frac{3}{-c}}(x-c t)\right), \quad c<0 .
\end{aligned}
$$

\section{Bifurcations of Phase Portraits of (4.2)}

Here we are considering a physical model where only bounded travelling waves are meaningful. So we only pay attention to the bounded solutions of (1.4). The bifurcation theory of dynamical systems (see [7-9]) plays an important role in our study.

$$
\text { For } a_{1}=-\frac{g}{c}, a_{2}=-1, a_{3}=\frac{1}{2 c}, \sigma=-c \text {, and } \epsilon=\frac{1}{c}, \text { Equation }
$$

becomes to

$$
u^{\prime \prime}(\xi)=-\frac{g}{c}-u(\xi)+\frac{1}{2 c} u^{2}(\xi)
$$


Equation (4.1) is equivalent to the two-dimensional systems as follows:

$$
\frac{d u}{d \xi}=y, \quad \frac{d y}{d \xi}=-\frac{g}{c}-u(\xi)+\frac{1}{2 c} u^{2}(\xi),
$$

with the first integral

$$
y^{2}=-\frac{2 g}{c}-u^{2}+\frac{1}{3 c} u^{3}+h,
$$

and

$$
H(u, y)=y^{2}+\frac{2 g}{c} u+u^{2}-\frac{1}{3 c} u^{3}=h .
$$

In this section, we study all possible periodic annuluses defined by the vector fields of (4.2) when the parameters $c, g$ are varied.

Denote that $\Delta=1+\frac{2 g}{c^{2}}$, which imply the relations in the $(c, g)$-parameter plane

$$
L: g=-\frac{1}{2} c^{2}
$$

Thus, we have

(i) For $\Delta=1+\frac{2 g}{c^{2}}>0$, there exist 2 equilibrium points of (4.2): $A_{ \pm}(c(1 \pm \sqrt{\Delta}), 0)$

(ii) For $\Delta=1+\frac{2 g}{c^{2}}<0$, there have no equilibrium points of (4.2).

Let $M\left(u_{e}, y_{e}\right)$ be the coefficient matrix of the linearized system of (4.2) at an equilibrium point $\left(u_{e}, y_{e}\right)$. Then, we have

$$
J\left(u_{i}, 0\right)=\operatorname{det}\left(M\left(u_{i}, 0\right)\right)=1-\frac{1}{c} u_{i} .
$$

By the theory of planar dynamical systems, we know that for an equilibrium point of a planar integrable system, if $J<0$, then the equilibrium point is a saddle point; if $J>0$ and $\operatorname{Trace}\left(M\left(u_{i}, y_{i}\right)\right)=0$, 
then it is a center point; if $J>0$ and $\operatorname{Trace}\left(M\left(u_{i}, y_{i}\right)\right)^{2}-4 J\left(u_{i}, y_{i}\right)>0$, then it is a node; if $J=0$ and the index of the equilibrium point is 0 , then it is a cusp, otherwise, it is a high order equilibrium point.

For the function defined by (4.4), we denote that

$$
h_{i}=H\left(u_{i}, 0\right)=-\frac{2 u_{i}}{3 c}\left[(c-3 g) u_{i}-2 g\right], \quad i=1,2 .
$$

We next use the above statements to consider the bifurcations of the phase portraits of (4.2). In the $(c, g)$-parameter plane, the curves $L$ and $c=0$ partition it into 4 shown in Figure 1.

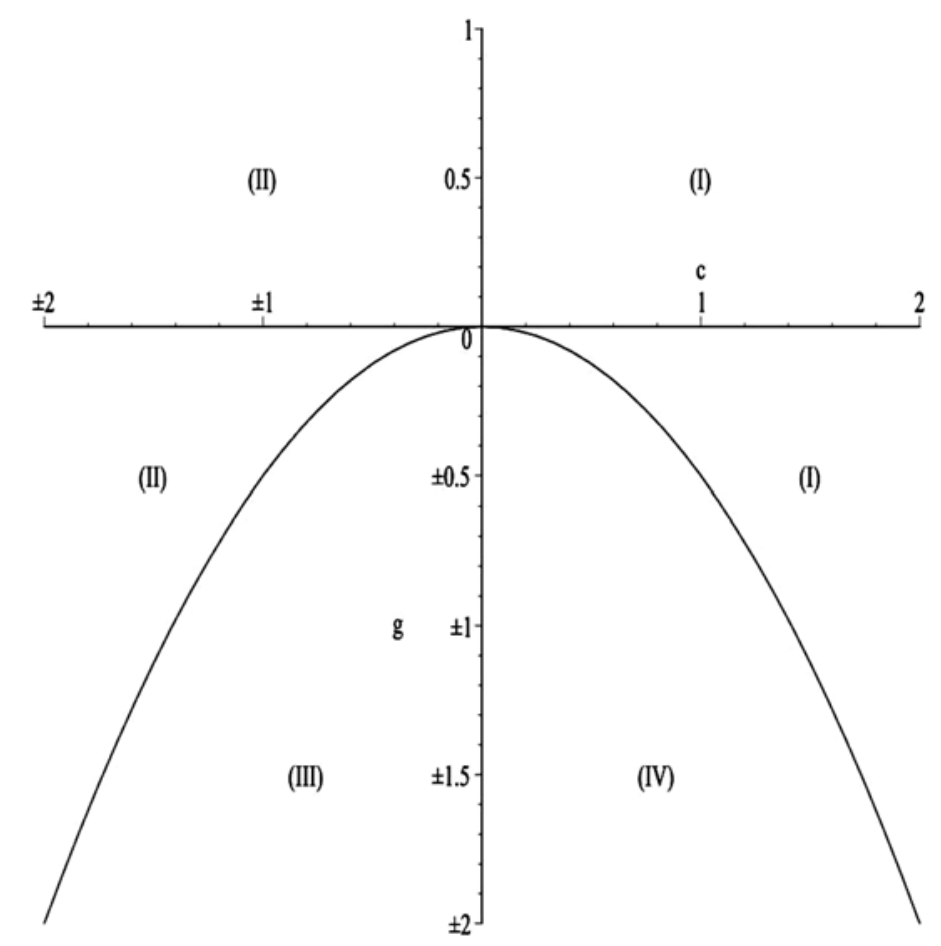

Figure 1. The bifurcation set of (4.2) in $(c, g)$-parameter plane. 
Figure 2 show the phase portraits of (4.2) for $\Delta>0$.

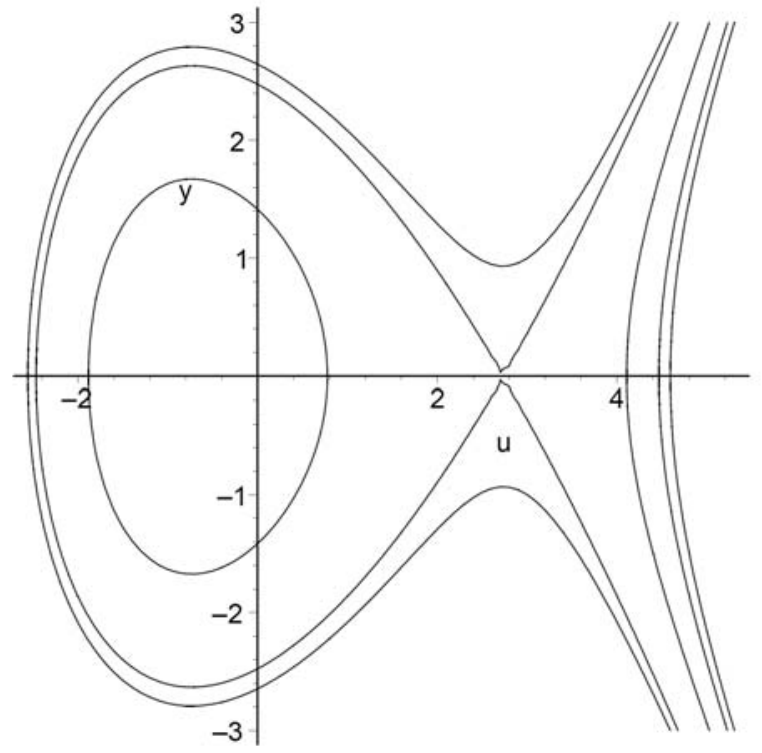

$(2-1)(c, g) \in(\mathrm{I})$

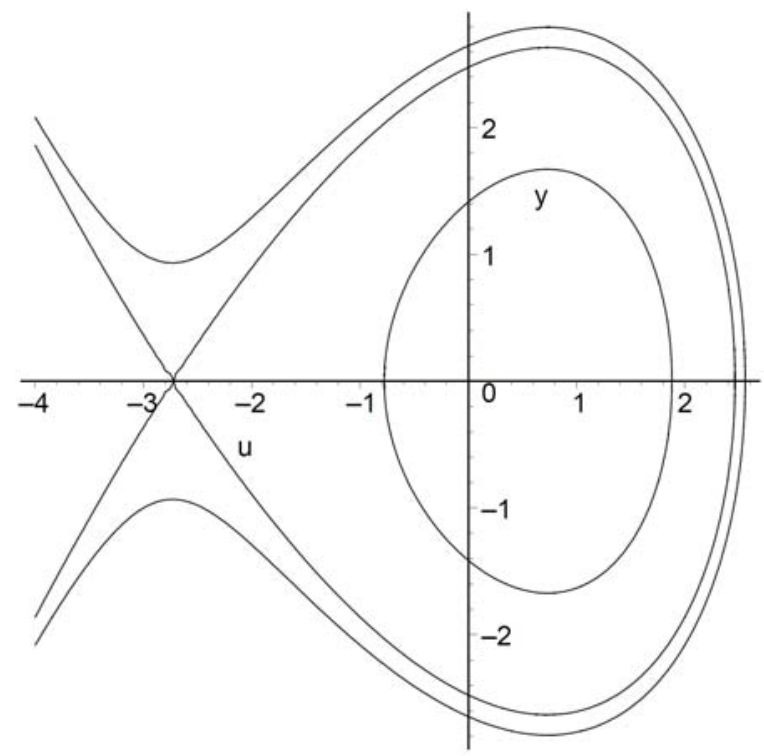

$(2-2)(c, g) \in(\mathrm{II})$ 


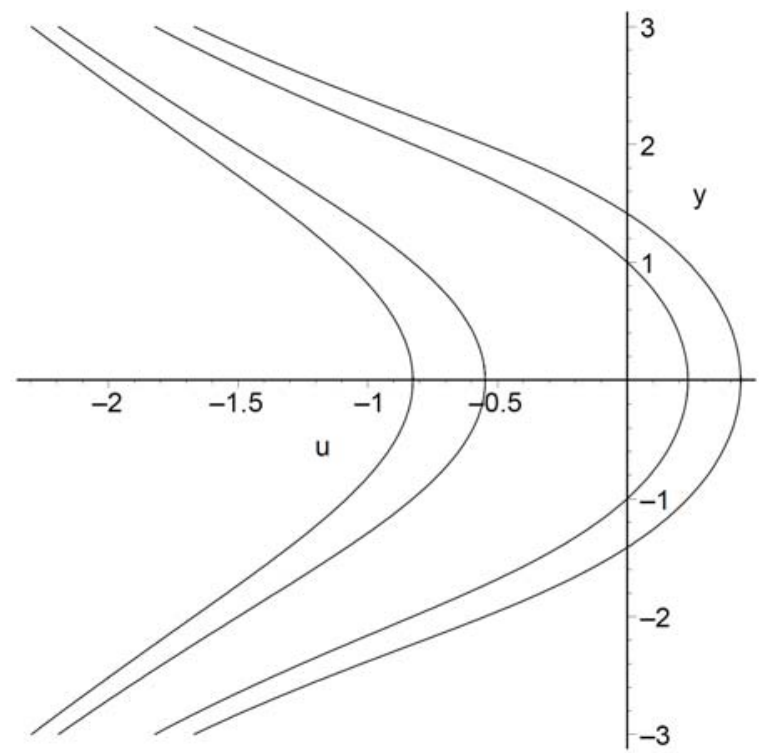

$(2-3)(c, g) \in(\mathrm{III})$

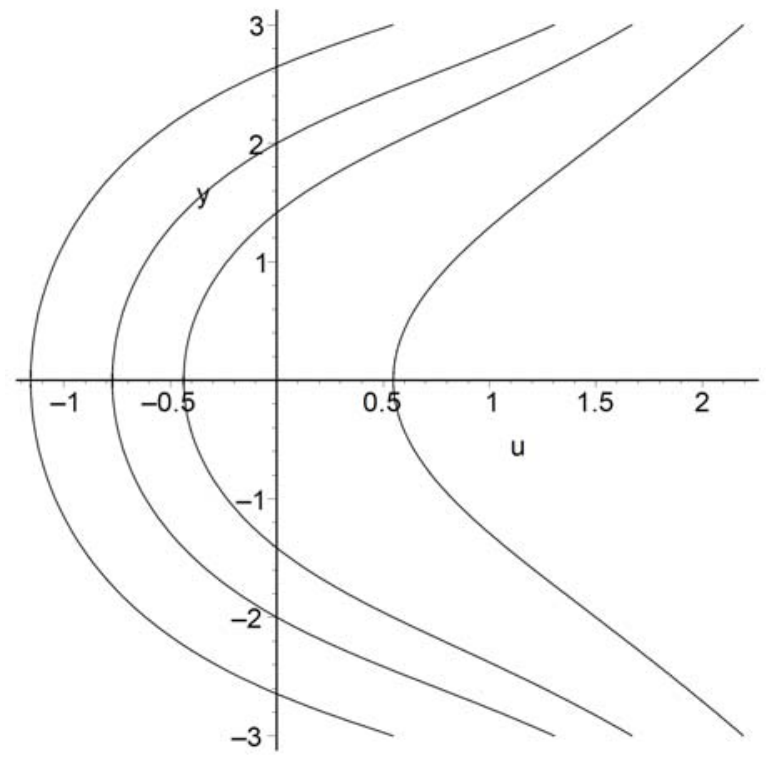

$(2-4)(c, g) \in(\mathrm{IV})$

Figure 2. The phase portraits of (4.2) for $\Delta>0$. 


\section{Exact Explicit Parametric Representations of Travelling wave Solutions of (1.1)}

5.1. Suppose that $(c, g) \in(\mathrm{I}), h_{1}=H\left(A_{+}, 0\right)=u_{+}\left(\frac{2 g}{c}+u_{+}-\frac{1}{3 c} u_{+}^{2}\right)$. In this case, we have the phase portrait of (4.1) shown in Figure 2 (2-1). We see from (4.2) that the arch curve connecting $A_{+}(c(1+\sqrt{\Delta}), 0)$ has the algebraic equation

$$
y^{2}=\frac{1}{3 c}\left(u-u_{+}\right)^{2}\left(u-u_{k}\right),
$$

where $u_{+}=c(1+\sqrt{\Delta}), u_{k}=2 u_{+}-3 c$. Thus, by using the first equation of (4.2) and (5.1), we obtain the parametric representation of this arch as follows:

$$
u_{5}(x, t)=u_{k}+\left(u_{+}-u_{k}\right) \tanh ^{2} \frac{1}{2} \sqrt{\frac{u_{+}-u_{k}}{3 c}}(x-c t) .
$$

Solution (5.2) gives rise to a smooth solitary wave solution of valley type of (1.1).

5.2. Suppose that $(c, g) \in(\mathrm{I}), h_{2}=H\left(A_{-}, 0\right), h \in\left(h_{1}, h_{2}\right)$. In this case, we have the phase portrait of (4.2) shown in Figure 2 (2-1). We see from (4.2) that arch curve around as the center $A_{-}\left(u_{-}, 0\right)$ has the algebraic equation

$$
y^{2}=\frac{1}{3 c}\left(u-u_{M}\right)\left(u-u_{l}\right)\left(u-u_{m}\right),
$$

where $u_{m}<0<u_{l}<u_{M}$. Thus, by using the first equation of (4.2) and (5.3), we obtain the parametric representation of this arch as follows:

$$
\begin{aligned}
& u_{6}(x, t)= \\
& \frac{u_{l}\left(u_{M}-u_{m}\right)-u_{M}\left(u_{l}-u_{m}\right) s n^{2}\left(\frac{1}{2} \sqrt{\frac{u_{M}-u_{m}}{3 c}}(x+y-c t), \sqrt{\frac{u_{l}-u_{m}}{u_{M}-u_{m}}}\right)}{u_{M}-u_{m}-\left(u_{l}-u_{m}\right) s n^{2}\left(\frac{1}{2} \sqrt{\frac{u_{M}-u_{m}}{3 c}}(x+y-c t), \sqrt{\frac{u_{l}-u_{m}}{u_{M}-u_{m}}}\right)},
\end{aligned}
$$


where $\operatorname{sn}(x, k)$ is the Jacobi elliptic functions with the modulo $k$ [10]. Solution (5.4) gives rise to a smooth periodic wave solutions of (1.1).

5.3. Suppose that $(c, g) \in(\mathrm{II}), h_{1}=H\left(A_{+}, 0\right)$. In this case, we have the phase portrait of (4.1) shown in Figure 2 (2-2). We see from (4.2) that the arch curve connecting $A_{+}(c(1+\sqrt{\Delta}), 0)$ has the algebraic equation

$$
y^{2}=-\frac{1}{3 c}\left(u-u_{+}\right)^{2}\left(u_{n}-u\right)
$$

where $u_{+}=c(1+\sqrt{\Delta}), u_{n}=3 c-2 u_{+}$. Thus, by using the first equation of (4.2) and (5.5), we obtain the parametric representation of this arch as follows:

$$
u_{7}(x, t)=u_{n}-\left(u_{n}-u_{+}\right) \tanh ^{2} \frac{1}{2} \sqrt{\frac{u_{n}-u_{+}}{-3 c}}(x-c t) .
$$

Solution (5.6) gives rise to a smooth solitary wave solution of peak type of (1.1).

5.4. Suppose that $(c, g) \in(\mathrm{II}), h_{2}=H\left(A_{-}, 0\right), h \in\left(h_{2}, h_{1}\right)$. In this case, we have the phase portrait of (4.2) shown in Figure $2(2-2)$. We see from (4.2) that arch curve around as the center $A_{-}\left(u_{-}, 0\right)$ has the algebraic equation

$$
y^{2}=\frac{1}{3 c}\left(u-\phi_{M}\right)\left(u-\phi_{l}\right)\left(u-\phi_{m}\right),
$$

where $\phi_{m}<\phi_{l}<0<\phi_{M}$. Thus, by using the first equation of (4.2) and (5.7), we obtain the parametric representation of this arch as follows:

$$
\begin{aligned}
& u_{8}(x, t)= \\
& \frac{\phi_{l}\left(\phi_{M}-\phi_{m}\right)-\phi_{M}\left(\phi_{l}-\phi_{m}\right) s n^{2}\left(\frac{1}{2} \sqrt{\frac{\phi_{M}-\phi_{m}}{3 c}}(x+y-c t), \sqrt{\frac{\phi_{l}-\phi_{m}}{\phi_{M}-\phi_{m}}}\right)}{\phi_{M}-\phi_{m}-\left(\phi_{l}-\phi_{m}\right) s n^{2}\left(\frac{1}{2} \sqrt{\frac{\phi_{M}-\phi_{m}}{3 c}}(x+y-c t), \sqrt{\frac{\phi_{l}-\phi_{m}}{\phi_{M}-\phi_{m}}}\right)} .
\end{aligned}
$$


Solution (5.8) gives rise to a smooth periodic wave solutions of (1.1).

\section{Discussion}

In this paper, we used the sine-cosine method and the method of dynamical bifurcation theory of the differentiable dynamics to study the generalized Kuramoto-Sivashinsky equation. The methods provided solitary wave solutions, solitary patterns wave solutions, and periodic wave solutions. Moreover, the obtained results in this work clearly demonstrate the reliability of the methods that were used.

\section{References}

[1] H. Aspe and M. C. Depassier, Evolution equation of surface waves in a convecting fluid, Phys. Rev. A 41 (1990), 3125-3128.

[2] A. J. Bernoff, Slowly varying fully nonlinear wave trains in the Ginzburg-Landau equation, Physica D 30 (1988), 363-381.

[3] E. J. Parkes and B. R. Duffy, An automated tanh-function method for finding solitary wave solutions to non-linear evolution equations, Comput. Phys. Commun. 98 (1996), 288-300.

[4] A. M. Wazwaz, A sine-cosine method for handling nonlinear wave equations, Math. Comput. Model. 40 (2004), 499-508.

[5] S. Q. Tang, Y. X. Xiao and Z. J. Wang, Travelling wave solutions for a class of nonlinear fourth order variant of a generalized Camassa-Holm equation, Appl. Math. Comput. 210 (2009), 39-47.

[6] J. B. Li, Singular Nonlinear Travelling Wave Equations: Bifurcations and Exact Solutions, Science Press, Beijing, 2013.

[7] S. N. Chow and J. K. Hale, Method of Bifurcation Theory, Springer-Verlag, New York, 1981.

[8] J. Guckenheimer and P. J. Holmes, Nonlinear Oscillations, Dynamical Systems and Bifurcations of Vector Fields, Springer-Verlag, New York, 1983.

[9] J. B. Li and H. H. Dai, On the Study of Singular Nonlinear Traveling Wave Equations: Dynamical System Approach, Science Press, Beijing, 2007.

[10] P. F. Byrd and M. D. Friedman, Elliptic Integrals for Engineers and Scientists, Springer-Verlag, New York, 1971. 